\title{
Pancreatic triacylglycerol distribution in type 2 diabetes. Reply to Hollingsworth K. G., Al Mrabeh A., Steven S. et al [letter]
}

\author{
Paul Begovatz ${ }^{1}$ - Alessandra Bierwagen ${ }^{1} \cdot$ Jesper Lundbom $^{1} \cdot$ Michael Roden $^{1,2}$
}

Received: 25 August 2015 / Accepted: 3 September 2015 / Published online: 23 September 2015

(C) Springer-Verlag Berlin Heidelberg 2015

Keywords Beta cell $\cdot$ Ectopic fat $\cdot$ Insulin sensitivity $\cdot$ Lipids · Magnetic resonance imaging $\cdot$ Pancreas $\cdot$ Pancreatic steatosis

\author{
Abbreviations \\ mDIXON Modified DIXON fat/water imaging sequence \\ LF-PRIDE Low-fat Philips Research Integrated \\ Development Environment
}

To the Editor: We welcome the comments of Hollingsworth et al [1], as the role of pancreas fat content in type 2 diabetes has become a hotly debated issue with diverse non-invasive measurement techniques providing seemingly contradictory results [2]. Although several fat/water imaging techniques have been proposed for assessing pancreatic fat content, including 2-point or multi-echo DIXON [3, 4] and fat-selective imaging techniques $[5,6]$, there is still no consensus or agreement on what constitutes a valid clinical measurement technique for non-invasive assessment of pancreatic fat. Here we respond to the issues raised and provide data comparing the 2-point 3D modified DIXON (mDIXON) method used in our publication [2] with the 3-point 2D DIXON method used by Hollingsworth et al [4]. We also demonstrate the importance of using noise bias correction when measuring pancreas fat.

Michael Roden

michael.roden@ddz.uni-duesseldorf.de

1 Institute for Clinical Diabetology, German Diabetes Center, Leibniz Center for Diabetes Research at Heinrich Heine University, Auf $m$ Hennekamp 65, 40225 Düsseldorf, Germany

2 Department of Endocrinology and Diabetology, Medical Faculty, Heinrich Heine University Düsseldorf, Düsseldorf, Germany
The first issue raised is that the 2-point mDIXON method yielded negative values for the fat fraction in the pancreas, which could indicate poor performance of the method. We have validated the 2-point mDIXON method in vitro using fat-water phantoms and in vivo by comparing with proton magnetic resonance spectroscopy ( $\left.{ }^{1} \mathrm{H}-\mathrm{MRS}\right)$ measurements [7]. In addition, as already demonstrated [2], the reproducibility of the 2-point mDIXON method was comparable to that of ${ }^{1} \mathrm{H}-\mathrm{MRS}$. The negative pancreas fat values are likely to be the result of using a noise bias correction algorithm called lowfat Philips Research Integrated Development Environment (LF-PRIDE) (Philips, Best, the Netherlands), which seemingly produces a more Gaussian noise distribution, which therefore results in statistically valid negative values. The noise distribution without noise bias correction is Rician, i.e. there are no negative values, which can lead to artificially high fat fractions when using DIXON techniques [8].

The second issue raised by Hollingsworth et al is that we reported no fat within pancreatic parenchymal tissue, which contradicts their findings of a $3.3-6.2 \%$ fat fraction in the pancreas of type 2 diabetic humans [1]. In our paper, we stated that the MRI measurements revealed regions of parenchymal tissue void of any relevant lipid accumulation in all participants, which can be taken as evidence against a uniform pancreatic steatosis. However, the pancreatic fat fraction of type 2 diabetic humans (obtained with the 2-point 3D mDIXON method) was up to $4 \%$ in some individuals [2]. Thus, it is misleading for Hollingsworth et al to claim that we suggest that there is no fat within the pancreatic parenchymal tissue. The lower pancreas fat fraction reported by us [2] compared Hollingsworth et al [1] is probably due to the noise bias, which does not seem to be corrected for in their method [4].

The third issue raised is that all measurements should have been carried out using a properly established 3-point DIXON technique to remove differences related to spectroscopic and 
imaging methods. As both ${ }^{1} \mathrm{H}$-MRS [9] and DIXON MRI [3-6] techniques have been used to measure pancreas fat previously, it was deemed appropriate to use both methods simultaneously. Furthermore, as previous studies have shown that ${ }^{1} \mathrm{H}-\mathrm{MRS}$ and MRI techniques may produce conflicting results for pancreatic fat measurements [9], the inclusion of both techniques in a study can provide additional clarity into the role of pancreas fat in type 2 diabetes. As all methods were analysed separately [2], the methodological differences are isolated and do not constitute a technical problem.

To clarify the seemingly contradictory results between our publication [2] and those of Hollingsworth et al [1], we measured pancreas fat content in three non-diabetic and three type 2 diabetic volunteers with both the 2-point 3D mDIXON [2] and the 3-point 2D DIXON [4] methods. Using the standard algorithm for calculation of fat fraction yielded similar values for the 2-point and the 3-point methods in both non-diabetic (mean \pm SEM: $5.45 \pm 0.57 \%$ [2-point] vs $6.35 \pm$ $0.50 \%$ [3-point]) and type 2 diabetic humans $(8.43 \pm 1.20 \%$ [2-point] vs $8.64 \pm 0.36 \%$ [3-point]). When using noise bias correction with the LF-PRIDE algorithm, the calculated pancreas fat was reduced in all participants, both in healthy (1.94 $\pm 0.59 \%$ [2-point] vs $1.22 \pm 0.68 \%$ [3-point]) and type 2 diabetic humans $(2.51 \pm 1.54 \%$ [2-point] vs $2.69 \% \pm 0.83 \%$ [3-point]). The different imaging methods and fat fraction calculation algorithms are illustrated for one representative volunteer in Fig. 1. These results highlight that without noise bias correction the resulting pancreas fat fraction can be
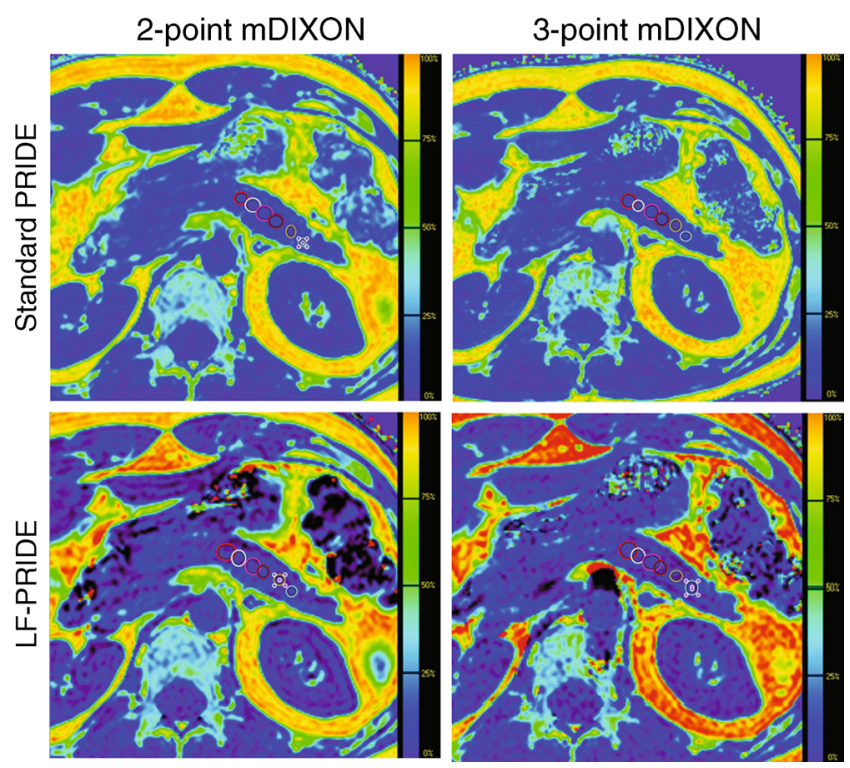

Fig. 1 Comparison of 2-point and 3-point mDIXON methods and the effects of noise bias correction. Colour-coded fat fraction maps for one volunteer, with the key showing per cent fat. The means of six regions of interest (ROIs; circles) were used to determine the fat fraction of the pancreas. 2-point mDixon: LF-PRIDE: $1.78 \pm 0.28 \%$; standard PRIDE: 5.97 $\pm 0.17 \%$; 3-point mDixon: LF-PRIDE: $1.49 \pm 0.25 \%$; standard PRIDE: $5.52 \pm 0.14 \%$. The results indicate that noise bias leads to overestimation of pancreatic fat in both 2-point and 3-point DIXON images overestimated by up to $5 \%$ points, in both the 2 -point and 3 -point methods.

Furthermore, our results are in line with current histological studies in human pancreases, as described in detail within the discussion of our publication [2]. Even though evidence for pancreatic intracellular fat accumulation has been found in animals $[10,11]$, other studies have failed to reproduce these results [12], and we have found no documented evidence of intracellular pancreatic fat accumulation in humans. As already mentioned, we do not claim that there is no fat in the parenchymal pancreas, and our results do not exclude the possibility of low levels of triacylglycerols [2]. Nevertheless, Pinnick et al [10] attributed the triacylglycerol content of the human pancreas to adipocyte infiltration, which was also independent of glycaemic status. In addition, a recent study using a multi-echo proton density fat fraction technique with noise bias correction also reported that pancreas fat was inhomogeneously distributed and independent of glycaemic status [13], further corroborating our findings.

In conclusion, noise bias can lead to an apparently high and even distribution of fat in the pancreas, which disappears after correction in both 2-point and 3-point DIXON images. We also found no difference in the performance of the 2-point and 3-point DIXON methods when measuring pancreas fat, indicating that methodological issues did not impair our measurement results or limit our conclusions. The role and regulation of adipocyte infiltration and intracellular pancreatic fat in the development of type 2 diabetes needs further research. Unfortunately, current non-invasive MRI and MRS techniques cannot distinguish between these two fat depots, which may or may not coexist within the pancreas, although our data support the view of a predominant role of adipose tissue infiltration to explain pancreatic triacylglycerol accumulation.

Acknowledgements The authors would like to thank A. Nagel and N. Achterath, Institute for Clinical Diabetology, German Diabetes Center, Düsseldorf, for taking care of the volunteers and help with the MRS/ MRI measurements.

Funding This work was supported by the Ministry of Science and Research of the State of North Rhine-Westphalia (MIWF NRW) and the German Federal Ministry of Health (BMG). This study was also supported in part by a grant from the Federal Ministry for Research (BMBF) to the German Center for Diabetes Research (DZD e.V.) and by the Helmholtz Alliance with Universities (Imaging and Curing Environmental Metabolic Diseases, ICEMED).

Duality of interest The authors declare that there is no duality of interest associated with this manuscript.

Contribution statement $\mathrm{PB}, \mathrm{AB}$ and JL carried out the measurements and analysis, and co-wrote the manuscript. MR conceived the study, cowrote the manuscript, and is the guarantor of this work. Lastly, all authors have provided final approval for the publication of this work. 


\section{References}

1. Hollingsworth KG, Al-Mrabeh A, Steven S, Taylor R (2015) Pancreatic triacylglycerol distribution in type 2 diabetes. Diabetologia. doi:10.1007/s00125-015-3718-1

2. Begovatz P, Koliaki C, Weber K et al (2015) Pancreatic adipose tissue infiltration, parenchymal steatosis and beta cell function in humans. Diabetologia 58:1646-1655

3. Sijens PE, Edens MA, Bakker SJ, Stolk RP (2010) MRIdetermined fat content of human liver, pancreas and kidney. World J Gastroenterol 16:1993-1998

4. Lim EL, Hollingsworth KG, Aribisala BS, Chen MJ, Mathers JC, Taylor R (2011) Reversal of type 2 diabetes: normalisation of beta cell function in association with decreased pancreas and liver triacylglycerol. Diabetologia 54:2506-2514

5. Schwenzer NF, Machann J, Martirosian P et al (2008) Quantification of pancreatic lipomatosis and liver steatosis by MRI: comparison of in/opposed-phase and spectral-spatial excitation techniques. Investig Radiol 43:330-337

6. Heni M, Machann J, Staiger $\mathrm{H}$ et al (2010) Pancreatic fat is negatively associated with insulin secretion in individuals with impaired fasting glucose and/or impaired glucose tolerance: a nuclear magnetic resonance study. Diabetes Metab Res Rev 26:200-205
7. Begovatz P, Koliaki C, Szendroedi J, Giani G, Bunke J, Roden M (2014) Pancreas fat via dual-echo mDIXON imaging shows that intracellular fat does not accumulate within the pancreas of healthy and T2DM subjects. Proc Int Soc Magn Reson 23:3608 (Abstract)

8. Reeder SB, Cruite I, Hamilton G, Sirlin CB (2011) Quantitative assessment of liver fat with magnetic resonance imaging and spectroscopy. J Magn Reson Imaging 34:spcone

9. Hu HH, Kim HW, Nayak KS, Goran MI (2010) Comparison of fatwater MRI and single-voxel MRS in the assessment of hepatic and pancreatic fat fractions in humans. Obesity (Silver Spring) 18:841847

10. Pinnick KE, Collins SC, Londos C, Gauguier D, Clark A, Fielding BA (2008) Pancreatic ectopic fat is characterized by adipocyte infiltration and altered lipid composition. Obesity (Silver Spring) 16: $522-530$

11. Lee Y, Lingvay I, Szczepaniak LS, Ravazzola M, Orci L, Unger RH (2010) Pancreatic steatosis: harbinger of type 2 diabetes in obese rodents. Int J Obes (Lond) 34:396-400

12. Navina S, Acharya C, DeLany JP et al (2011) Lipotoxicity causes multisystem organ failure and exacerbates acute pancreatitis in obesity. Sci Transl Med 3:107ra110

13. Kuhn JP, Berthold F, Mayerle J et al (2015) Pancreatic steatosis demonstrated at MR imaging in the general population: clinical relevance. Radiology 276:129-136 